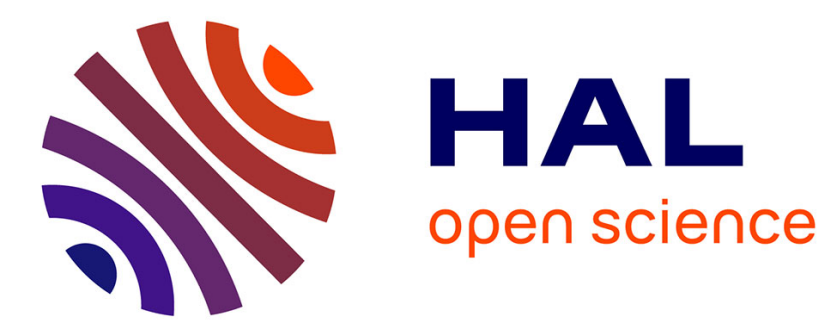

\title{
How to score alternatives when criteria are scored on an ordinal scale
}

\author{
Michel Grabisch
}

\section{To cite this version:}

Michel Grabisch. How to score alternatives when criteria are scored on an ordinal scale. Journal of Multi-Criteria Decision Analysis, 2008, 15 (1-2), pp.31-44. 10.1002/mcda.422 . halshs-00340381

\section{HAL Id: halshs-00340381 https://shs.hal.science/halshs-00340381}

Submitted on 20 Nov 2008

HAL is a multi-disciplinary open access archive for the deposit and dissemination of scientific research documents, whether they are published or not. The documents may come from teaching and research institutions in France or abroad, or from public or private research centers.
L'archive ouverte pluridisciplinaire HAL, est destinée au dépôt et à la diffusion de documents scientifiques de niveau recherche, publiés ou non, émanant des établissements d'enseignement et de recherche français ou étrangers, des laboratoires publics ou privés. 


\title{
How to score alternatives when criteria are scored on an ordinal scale
}

\author{
Michel GRABISCH \\ Université de Paris I - Panthéon-Sorbonne \\ Centre d'Economie de la Sorbonne \\ 106-112 Bd. de l'Hôpital, 75013 Paris, France \\ Tel (+33) 1-44-07-82-85, Fax (+33) 1-44-07-83-01 \\ email michel.grabisch@univ-paris1.fr
}

\begin{abstract}
We address in this paper the problem of scoring alternatives when they are evaluated with respect to several criteria on a finite ordinal scale $E$. We show that in general, the ordinal scale $E$ has to be refined or shrunk in order to be able to represent the preference of the decision maker by an aggregation operator belonging to the family of mean operators. The paper recalls previous theoretical results of the author giving necessary and sufficient conditions for a representation of preferences, and then focusses on describing practical algorithms and examples.
\end{abstract}

Keywords: ordinal scale, aggregation of scores, mean operator, refinement of scale 


\section{Introduction}

Every teacher has in his life encountered the following problem:

Given a set of students who are evaluated on a finite common ordinal scale (say, from $\alpha=$ excellent to $\epsilon=$ dreadful) for the different subjects (say, mathematics, economics, foreign languages, etc.), how to compute an overall score for each student?

An obvious answer to this problem is to convert the labels of the ordinal scale into numbers (say, from 5 to 1), and to use standard arithmetic or weighted average to compute the overall score. Eventually, this numerical score could be converted back to the ordinal scale, if the average score happens by chance to be equal to one of the chosen numbers for the labels, or if it is not the case, to introduce some arbitrary additional labels on the ordinal scale, like $\alpha^{-}$or $\beta^{+}$.

Although we believe that by far this is common practice, we know from measurement theory (see, e.g., Roberts [1979]) that such a method is meaningless, in the sense that, depending on which numbers are chosen, provided their ordering reflect the ordering of labels, the ranking of the students may change, a situation which is not acceptable. We illustrate this by the following example.

EXAmple 1: We consider the ordinal scale $\alpha>\beta>\gamma>\delta>\epsilon$, three subjets $(\mathrm{M}=$ mathematics, $\mathrm{E}=$ economics, $\mathrm{L}=$ language $)$, and three students $a, b, c$, having the following scores:

\begin{tabular}{|c|c|c|c|}
\hline student & $\mathrm{M}$ & $\mathrm{E}$ & $\mathrm{L}$ \\
\hline$a$ & $\alpha$ & $\gamma$ & $\gamma$ \\
$b$ & $\beta$ & $\beta$ & $\beta$ \\
$c$ & $\delta$ & $\beta$ & $\alpha$ \\
\hline
\end{tabular}

Let us consider 4 different ways to convert the ordinal scale into numbers:

- scale 1 : $\alpha=10, \beta=7.5, \gamma=5, \delta=2.5, \epsilon=0$

- scale 2 : $\alpha=10, \beta=7, \gamma=5, \delta=2, \epsilon=0$

- scale 3: $\alpha=10, \beta=6, \gamma=5, \delta=2, \epsilon=0$

- scale $4: \alpha=10, \beta=6, \gamma=4, \delta=2, \epsilon=0$.

Then the average scores are:

\begin{tabular}{|c|c|c|c|c|}
\hline student & scale 1 & scale 2 & scale 3 & scale 4 \\
\hline$a$ & 6.67 & 6.67 & 6.67 & 6 \\
$b$ & 7.5 & 7 & 6 & 6 \\
$c$ & 6.67 & 6.33 & 6 & 6 \\
\hline
\end{tabular}

Clearly, all 4 rankings are different, even for such small variations in the conversion of the scale. 
For this reason, we adopt in this paper a purely ordinal approach, avoiding any numerical conversion.

There are of course many methods in multicriteria decision making able to deal with ordinal scores (see, e.g., Pomerol and Barba-Romero [2000] for a large survey of MCDA methods): it is the case for the lexicographic method and its variants (lexicographic semiorder of Pirlot and Vincke [Pirlot and Vincke, 1992], lexicographic permutation method of Massam and Askew [Massam and Askew, 1982]), and in principle of all outranking methods like ELECTRE [Roy, 1968, 1973], PROMETHEE [Brans and Vincke, 1985], TACTIC [Vansnick, 1986], etc., and permutation methods, as QUALIFLEX of Paelinck [Paelinck, 1976]. All these methods however produce as final result an order on the alternatives, and not a score. There exists however a few methods able to produce a score from ordinal scores: these are for example the MACBETH approach of Bana e Costa and Vansnick [Bana e Costa and Vansnick, 1994, 1997], the TOMASO method of Marichal, Meyer and Roubens [Meyer and Roubens, 2005, Marichal et al., 2005b], and the rule-based approach of Greco, Matarazzo and Słowiński [Greco et al., 2005]. The MACBETH approach maps ordinal scores on a numerical scale in a way which is consistent with measurement theory, but it requires from the decision maker an intensity of preference, not only a (binary) preference. The TOMASO approach basically uses the number of times an alternative beats (or is beaten by) other alternatives for a given criterion, and this numerical information is fed into a Choquet integral (a generalization of the weighted sum). The problem is then that the final score is on a scale which has no relation with the original scale of scores. Lastly, the decision rule approach is not properly a method providing scores, but rather it maps alternatives to some predefined ordered classes, which could be considered as scores, but they have to be predefined, contrary to our approach.

Our aim is to address the scoring problem in the case where scores are expressed on some finite ordinal scale $E$, and as the above overview shows, no proper method seems to exist for the time being. It could be seen as a purely aggregation problem, and it is then natural to impose commonly accepted properties for aggregation, such as various kinds of nondecreasingness, internality, etc., thus making it similar to what is called in the numerical case a mean operator. However, we want to address the problem in a decision theoretic framework, which means that our first aim is to faithfully represent the revealed preferences of the decision maker, in the sense that alternative $a$ is at least as good as alternative $b$ if and only if the overall score of $a$ is greater or equal to the overall score of $b$.

In the domain of aggregation functions, few studies have been done on finite scales, and to the knowledge of the author, almost none deals with preference representation. Conjunctive and disjunctive (hence not internal) aggregation functions on finite scales have been studied in detail by Fodor [2000], and by Mas, Torrens et al. [Mas et al., 1999, 2003]. Their result are limited since they consider aggregation functions as mappings from $E^{n}$ to $E$, where $E$ is some finite scale, which is obviously very limitative as subsequent examples will show. On mean operators on finite scales, there exists a fundamental paper by Ovchinnikov [Ovchinnikov, 1996], followed by Marichal and Mesiar [Marichal and Mesiar, 2004, Marichal et al., 2005a], but their point of view is rather different (although complementary of ours) since they are not concerned with preference representation, but with the meaningfulness of means, in the measurement theoretic sense. Concerning this 
last topic, Rico et al. [2005] have given necessary and sufficient conditions for the representation of preference by a Sugeno integral [Sugeno, 1974, Marichal, 2000], a particular class of mean operators. Again, their results are limitative since $E$ is considered to be fixed.

The problem of finding all mean operators representing the preferences of a decision maker on a set of alternatives whose scores are given on some common ordinal scale, has been recently solved by the author in its full generality [Grabisch, 2006]. The aim of this paper is to present the practical side of this theoretical work, in the framework of multicriteria decision making, which seems to be the most appropriate. We also adopt here a more readable notation, although less concise.

In Section 2, we clearly state the problem we want to address and introduce necessary notations and concepts. Section 3 summarizes the main theoretical results of Grabisch [2006], and Section 4 gives practical algorithms solving the problem, and an example illustrating previous results and algorithms. Section 5 gives some final remarks.

\section{Statement of the problem and notations}

\subsection{Basic notations}

We consider alternatives evaluated on a set $N$ of $n$ criteria. All evaluations are expressed on a finite ordinal scale $E$ of $k$ elements $e_{1}<e_{2}<\cdots<e_{k}$, assuming that commensurability problems among criteria have been solved beforehand. Thus, a given alternative $a$ is identified with the vector $\left(a_{1}, \ldots, a_{n}\right) \in E^{n}$ of its scores, and we consider in the sequel a set $A \subseteq E^{n}$ of alternatives. As usual, for $a, b \in A, a \leq b$ stands for $a_{i} \leq b_{i}, i=1, \ldots, n$.

We assume that the decision maker can express his/her preferences on $A$ under the form of a weak order (transitive and complete) $\succsim$ on $A \times A$. We denote as usual $a \sim b$ if $a \succsim b$ and $b \succsim a$ hold, and $a \succ b$ if $a \succsim b$ and $\neg(b \succsim a)$.

We denote by $A_{1}, \ldots, A_{k^{\prime}}$ the equivalence classes of $\sim$, i.e., $\forall a, b \in A_{i}, a \sim b$, for $i=1, \ldots, k^{\prime}$, which we call indifference classes. We number them in such a way that $\forall a \in A_{i}, \forall a^{\prime} \in A_{j}, a \succ a^{\prime} \Leftrightarrow i>j$.

We call $(A, \succsim, E)$ the decision profile of the decision maker. Throughout the paper, we will assume non triviality of $\succsim$, i.e., there exist $a, b \in A$ such that $a \succ b$.

We introduce useful notations about indifference classes, alternatives and intervals of $E$. First, open intervals are denoted like $] e_{i}, e_{j}\left[\right.$. For a given closed interval $I=\left[e_{i}, e_{j}\right]$, left and right bounds are denoted by $\operatorname{left}(I):=e_{i}$ and $\operatorname{right}(I):=e_{j}$ respectively. Given two closed intervals $I:=\left[e_{i}, e_{j}\right]$ and $I^{\prime}=\left[e_{i^{\prime}}, e_{j^{\prime}}\right]$ such that $j \leq i^{\prime}$, the inner and outer distances between $I$ and $I^{\prime}$ are respectively defined by:

$$
\begin{aligned}
d\left(I, I^{\prime}\right) & :=i^{\prime}-j \\
D\left(I, I^{\prime}\right) & :=j^{\prime}-i .
\end{aligned}
$$

The inner distance is assumed to be 0 for intersecting intervals. The outer distance is 0 if and only if both intervals coincide and are reduced to a singleton. The cardinal of some interval $I=\left[e_{i}, e_{j}\right]$ is the number of elements in $I$, denoted by $|I|:=j-i+1$.

For a given alternative $a \in A$, the span of $a$ is the interval

$$
\operatorname{span}(a):=\left[\min _{i=1}^{n} a_{i}, \max _{i=1}^{n} a_{i}\right]
$$


We can extend this definition to indifference classes in the obvious way: for any indifference class $A_{j}$, we define its span as the interval

$$
\operatorname{span}\left(A_{j}\right):=\left[\min _{a \in A_{i}} \min _{j=1}^{n} a_{j}, \max _{a \in A_{i}} \max _{j=1}^{n} a_{j}\right] .
$$

Note that in general, $\operatorname{span}\left(A_{j}\right)$ is not the union of spans of its members. Since $\operatorname{span}(a)$ is an interval, we can consider left $(\operatorname{span}(a))$ and right $(\operatorname{span}(a))$, which we will denote with some abuse by left $(a)$ and $\operatorname{right}(a)$ (similarly for $\operatorname{left}\left(A_{j}\right), \operatorname{right}\left(A_{j}\right)$ ).

In order to avoid cumbersome conditions for some definitions, we introduce two (fictitious) additional elements $e_{0}$ and $e_{k+1}$ in $E$, such that $e_{0}<e_{1}$ and $e_{k}<e_{k+1}$, and the fictitious classes $A_{0}$ and $A_{k^{\prime}+1}$ (worst and best possible classes), with $\operatorname{span}\left(A_{0}\right):=\left\{e_{0}\right\}$, and $\operatorname{span}\left(A_{k^{\prime}+1}\right)=\left\{e_{k+1}\right\}$.

\subsection{The representation problem}

Our aim is to find a representation of $\succsim$ by a mapping $u$ from $E^{n}$ to some ordinal scale $C$ endowed with a total order $\leq$, such that $a \succsim b$ if and only if $u(a) \geq u(b)$. The finiteness of $E$ makes the task difficult, and as it will become clear, in many cases, $C$ cannot be taken equal to $E$.

To settle our requirements on the kind of aggregation, let us take at first $C=E$, supposing we have in $E$ as many degrees as we want, and consider an aggregation function $G: E^{n} \rightarrow E$ which should represent $\succsim$ (hence $u=G$ ). In multicriteria decision aid, the following requirements on $G$ are common practice.

Definition 1 Let $G: E^{n} \longrightarrow E$. We say that $G$ is:

(0) weakly monotone if $\forall a, a^{\prime} \in E^{n}$, left $(a) \geq \operatorname{right}\left(a^{\prime}\right)$ implies that $G(a)<G\left(a^{\prime}\right)$ cannot occur.

(i) internal if $\forall a \in E^{n}, G(a) \in \operatorname{span}(a)$;

(ii) non decreasing if $\forall a, a^{\prime} \in E^{n}, a \geq a^{\prime}$ implies $G(a) \geq G\left(a^{\prime}\right)$;

(iii) unanimously increasing if it is non decreasing, and $\forall i \in\{1, \ldots, n\}, a_{i}>a_{i}^{\prime}$ implies $G(a)>G\left(a^{\prime}\right)$

(iv) (strictly) increasing if it is non decreasing, and $\forall a, a^{\prime} \in E^{n}, a \geq a^{\prime}$ and $a_{i}>a_{i}^{\prime}$ for at least one $i \in\{1, \ldots, n\}$ imply $G(a)>G\left(a^{\prime}\right)$.

The operator is said to be a mean in the weak sense or a weak mean operator for short if only (i) holds, a mean operator if (i) and (ii) hold, a unanimously increasing mean if (i) and (iii) hold, and a strict mean if (i) and (iv) hold.

Internality means that the overall evaluation should not be beyond the range of the scores, while non decreasingness ensures that an improvement of one score cannot decrease the overall score. Note that internality implies weak monotonicity, but not the converse.

The different types of mean operators are closely related to the following properties of the decision profile, as it will be shown later. 
Definition 2 Let $A \subseteq E^{n}$ and $\succsim$ be a weak order on $A$. The decision profile $(A, \succsim, E)$ is

(i) weakly coherent if there is no $a, b$ in $A$ such that $a \succ b$ and $\operatorname{right}(a) \leq \operatorname{left}(b)$;

(ii) coherent if for no pair of alternatives $a, b$, we have both $a \succ b$ and $a \leq b$ (or equivalently, $a \geq b$ implies $a \succsim b$ );

(iii) strongly coherent if it is coherent, and for $a, b \in A, a_{i}>b_{i}$ for $i=1, \ldots, n$ implies $a \succ b$;

(iv) strictly coherent if for $a, b \in A, a \geq b$ and $a_{i}>b_{i}$ for at least one $i$ in $\{1, \ldots, n\}$, imply $a \succ b$.

Obviously, strict coherence implies strong coherence, which implies coherence, which in turn implies weak coherence. Remark that if we impose only coherence, we may have $a$ strictly greater than $b$ and $a \sim b$. Conditions (ii), (iii) and (iv) are sometimes called monotonicity, weak Pareto and strong Pareto conditions respectively.

We come back to the case where $E$ has a limited number of degrees, and let us show that the weakest requirement on $G$, which is to be internal (weak mean operator) has two important consequences:

- the number of degrees in $E$ may be insufficient. To see this, we consider again the example of students, with a scale from $e_{1}$ to $e_{5}$, and two subjects (mathematics and language):

\begin{tabular}{|c|c|c|}
\hline student & mathematics & language \\
\hline$a$ & $e_{5}$ & $e_{5}$ \\
$b$ & $e_{5}$ & $e_{4}$ \\
$c$ & $e_{4}$ & $e_{5}$ \\
$d$ & $e_{5}$ & $e_{3}$ \\
\hline
\end{tabular}

Assume the preference relation is $a \succ b \succ c \sim d$. This preference is plausible, since $a$ dominates $b, b$ is preferred to $c$ because mathematics is considered more important than language, and $c \sim d$ because there is compensation. Internality forces to give $e_{5}$ as overall score to $a$, and $e_{5}$ or $e_{4}$ to $b$ and $c$. But then it is impossible to represent the preference since only for $a, b, c$ we need three different degrees. A solution is to make a refinement of the scale by inserting new degrees. For example, defining $C:=\left\{e_{1}<e_{2}<e_{3}<e_{4}<e_{4}^{\prime}<e_{5}\right\}$ solves the problem, since we could assign $e_{4}^{\prime}$ to $b$, and $e_{4}$ to $c$ and $d$.

- the number of degrees in E may be unnecessarily large. We consider the following example.

EXAMPLE 2: A consumer is asked to espress his/her preference on four cars $a, b, c, d$, knowing their scores on two qualitative criteria, say comfort and safety. The qualitative scale has five degrees: $e_{1}=$ very bad, $e_{2}=$ bad, $e_{3}=$ average, $e_{4}=$ good, and $e_{5}=$ very good. 


\begin{tabular}{|c|c|c|}
\hline car & criterion 1 & criterion 2 \\
\hline$a$ & $e_{1}$ & $e_{1}$ \\
$b$ & $e_{2}$ & $e_{2}$ \\
$c$ & $e_{3}$ & $e_{5}$ \\
$d$ & $e_{5}$ & $e_{4}$ \\
\hline
\end{tabular}

The preference given is $a \sim b \prec c \prec d$, so that the profile is coherent. Although $b$ dominates $a$, the preference $a \sim b$ can be explained by the fact that cars $a$ and $b$ are so bad that the consumer does not feel necessary to distinguish them. Although there are several solutions for $b, c, d$ (e.g., $G(b)=e_{2}, G(c)=e_{3}$ and $\left.G(d)=e_{4}\right)$ which are internal, no internal function $G$ can represent the indifference between $a$ and $b$.

The above example can nevertheless be solved if one "shrinks" elements $e_{1}$ and $e_{2}$ into a single one, say $e_{1}^{\prime}$. Denoting the shrunk scale by $E^{\prime}$ and the shrinking operation by $f: E \longrightarrow E^{\prime}$, a representation on $E^{\prime}$ becomes possible through the function $f \circ G$, taking $G$ as above, and $G(a)=e_{1}, G(b)=e_{2}$. Note that $E^{\prime}$ is nothing else than a partition of $E$. This situation can arise if the scale $E$ contains elements which are in fact indiscernible or non significant for the decision maker.

Summarizing the above discussion, the problem we want to address in its full generality can be formulated as follows:

Given a decision profile $(A, \succsim, E)$, with $E$ being a finite ordinal scale, under which conditions is it possible to find a representation under the form $u=$ $f \circ G$, with $f: E \longrightarrow E^{\prime}$ a non decreasing mapping defining a partition $E^{\prime}$ of $E$, and $G: E^{n} \longrightarrow E$ is a (weak, strong, strict) mean operator? In case of impossibility, would a refinement of $E$ solve the problem?

\section{Theoretical results}

The following concepts play a central role in the sufficient and necessary conditions for representation.

Definition 3 Let $(A, \succsim, E)$ be a decision profile, and $A_{j}$ be some indifference class of $\succsim$, $j \in\left\{1, \ldots, k^{\prime}\right\}$. The core of $A_{j}$ is defined as:

$$
K_{j}:= \begin{cases}{\left[\min _{a \in A_{j}} \operatorname{right}(a), \max _{a \in A_{j}} \operatorname{left}(a)\right]} & \text { if } \min _{a \in A_{j}} \operatorname{right}(a) \leq \max _{a \in A_{j}} \operatorname{left}(a) \\ \emptyset & \text { otherwise. }\end{cases}
$$

The core is non empty every time there exist two alternatives $a, b$ in $A_{j}$ with disjoint spans (or coinciding on only one point), i.e., such that $\min _{i} a_{i} \geq \max _{i} b_{i}$. Figure 1 illustrates the definition with threee situations involving three alternatives $a, b, c$ belonging to the same class, on a 7-elements scale. On the left, there is no pair of alternatives with disjoint spans or coinciding on only one point, so that the core of this class is empty. In the center, the intersection of spans of $a$ and $c$ is $\left\{e_{3}\right\}$, hence the core is $\left\{e_{3}\right\}$. On the right, the core is caused by $a$ and $c$ which have disjoint spans. Note that if a class $A_{j}$ is such 


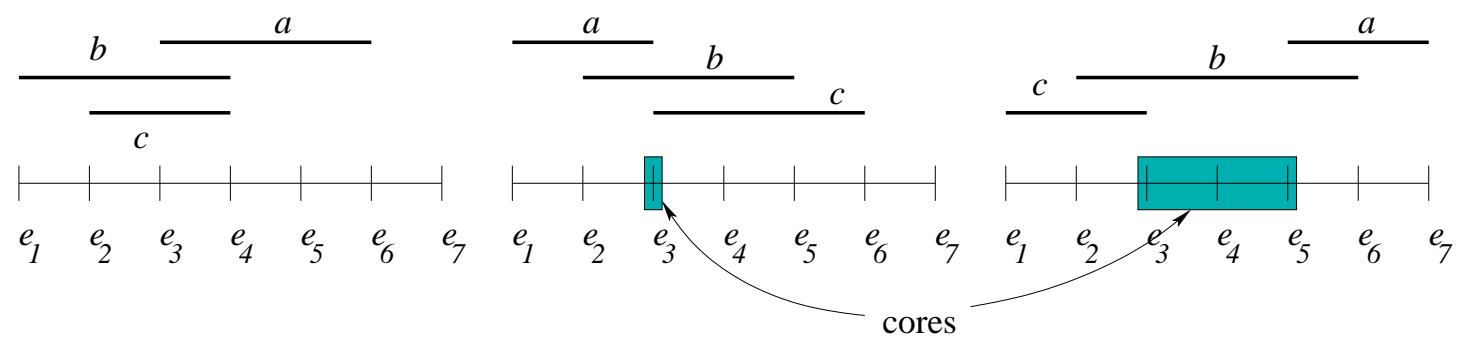

Figure 1: The core of a class: (left) empty core, (center and right) non empty core

that $\operatorname{span}\left(A_{j}\right)=\left\{e_{l}\right\}$, then its core is its span, i.e. $K_{j}=\left\{e_{l}\right\}$. In particular, this is always the case for the fictitious classes $A_{0}$ and $A_{k^{\prime}+1}$.

Definition 4 Let $(A, \succsim, E)$ be a decision profile. For any indifference class $A_{j}, j=$ $1, \ldots, k^{\prime}$, the interior of $A_{j}$ is the interval defined by:

$$
\left.\AA_{j}:=\operatorname{span}\left(A_{j}\right) \cap\right] \max _{a \prec A_{j}} \operatorname{left}(a), \min _{a \succ A_{j}} \operatorname{right}(a)[
$$

where, with some abuse of notations, $a \prec A_{j}$ means any alternative ranked strictly lower than any alternative in $A_{j}$, otherwise said, $a \in \bigcup_{j^{\prime}<j} A_{j^{\prime}}$ (and similarly for $a \succ A_{j}$ ).

Figure 2 illustrates the definition, with three classes, and $a, b, c \in A_{1}, d, e$ in $A_{2}$, and $f, g, h \in A_{3}$. The interior of a class indicates the allowable range for scores of any alternative of that class. For example, in Figure 2, $e_{9}$ is not in the interior of $A_{2}$, otherwise there would be no available degree in $E$ for the score of $h$, ranked higher than alternatives of class $A_{2}$. Subsequent theorems 2 and 3 will indeed show that the nonemptiness of the interiors is a necessary condition for the existence of a representation. Note that

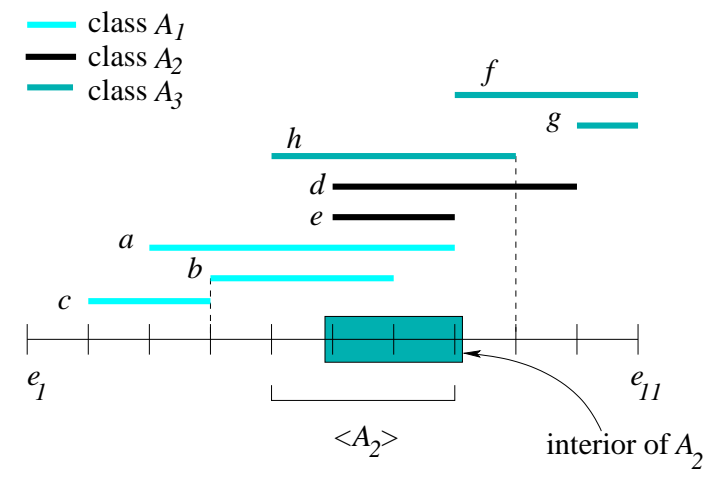

Figure 2: Interior of class $A_{2}$. The notation $\left\langle A_{2}\right\rangle$ means $\max _{a \prec A_{2}} \operatorname{left}(a), \min _{a \succ A_{2}} \operatorname{right}(a)[$.

$\min _{a \succ A_{k^{\prime}}} \operatorname{left}(a)=e_{k}$ and $\max _{a \prec A_{1}} \operatorname{right}(a)=e_{1}$, thanks to the additional classes $A_{0}$ and $A_{k^{\prime}+1}$. 


\subsection{Representation without a refinement of the scale}

(see Grabisch [2006] for proofs of these results)

Theorem 1 Let $(A, \succsim, E)$ be a decision profile with $k^{\prime}$ indifference classes. It exists a representation of $(A, \succsim, E)$ by $f \circ G$, where $G: E^{n} \longrightarrow E$ is a weak mean operator (resp. a mean operator, a strong mean, a strict mean), and $f$ defines a partition of $E$, if and only if the following conditions are satisfied:

(i) $(A, \succsim, E)$ is weakly coherent (resp. coherent, strongly coherent, strictly coherent)

(ii) $\forall j, j^{\prime} \in\left\{0, \ldots, k^{\prime}+1\right\}$ such that $j>j^{\prime}$ and $K_{j}, K_{j^{\prime}} \neq \emptyset$,

$$
d\left(K_{j^{\prime}}, K_{j}\right) \geq j-j^{\prime}
$$

(iii) $\AA_{j} \neq \emptyset$ for $j=1, \ldots, k^{\prime}$.

(iv) $\forall j, j^{\prime} \in\left\{1, \ldots, k^{\prime}\right\}$ such that $j>j^{\prime}$,

$$
D\left(\AA_{j^{\prime}}, \AA_{j}\right) \geq j-j^{\prime} .
$$

Figure 3 illustrates the theorem. We consider 8 alternatives $a, b, c, d, e, f, g, h, 3$ criteria and a scale $E$ with 9 elements. The decision profile is defined as follows.

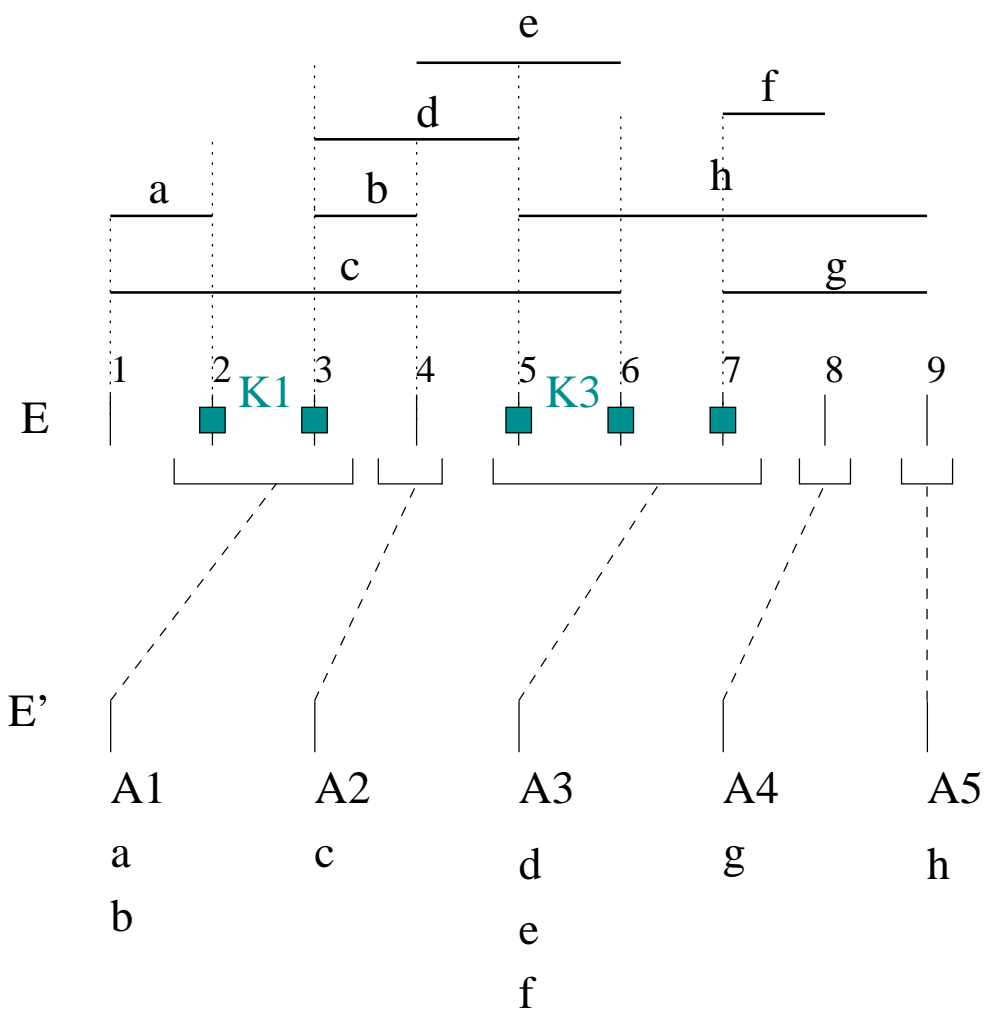

Figure 3: An example of preference representation 


\begin{tabular}{|c|c|c|c|c|}
\hline alternative & criterion 1 & criterion 2 & criterion 3 & class \\
\hline$a$ & $e_{1}$ & $e_{2}$ & $e_{2}$ & $A_{1}$ \\
$b$ & $e_{4}$ & $e_{3}$ & $e_{3}$ & $A_{1}$ \\
$c$ & $e_{1}$ & $e_{1}$ & $e_{6}$ & $A_{2}$ \\
$d$ & $e_{3}$ & $e_{5}$ & $e_{4}$ & $A_{3}$ \\
$e$ & $e_{4}$ & $e_{4}$ & $e_{6}$ & $A_{3}$ \\
$f$ & $e_{8}$ & $e_{7}$ & $e_{7}$ & $A_{3}$ \\
$g$ & $e_{9}$ & $e_{8}$ & $e_{7}$ & $A_{4}$ \\
$h$ & $e_{5}$ & $e_{9}$ & $e_{7}$ & $A_{5}$ \\
\hline
\end{tabular}

The preference is $a \sim b \prec c \prec d \sim e \sim f \prec g \prec h$, hence the classes $A_{1} \prec A_{2} \prec \cdots \prec A_{5}$. There are two nonempty cores $K_{1}=\left\{e_{2}, e_{3}\right\}$ and $K_{3}=\left\{e_{5}, e_{6}, e_{7}\right\}$, the other ones are empty. It can be verified that the profile is coherent, and conditions (ii), (iii) and (iv) of the theorem are satisfied. Hence there exists a mean operator $G$ and a partition of $E$ representing the preference. The finest partition is indicated by the brackets. Note that $e_{1}$ is not used in the representation. Solutions for $G$ are: $G(a)=e_{2}, G(b)=e_{3}$, $G(c)=e_{4}, G(d)=e_{5}, G(e)=e_{5}$ or $e_{6}, G(f)=e_{7}, G(g)=e_{8}$ and $G(h)=e_{9}$. Note that even if $a \sim b$, it is possible to have $G(a)=e_{2}$ and $G(b)=e_{3}$ since elements $e_{2}$ and $e_{3}$ are shrunk into a single one.

Let us comment briefly about the conditions (i) to (iv). Condition (i) is a translation of the fact that $G$ is some type of mean operator. Hence no mean operator can represent the preference if (i) is not satisfied. Condition (iii) says that the interior of classes should not be empty. As explained above, the interior of the class is the allowable range for putting overall scores of alternatives of that class, considering the minimal range taken by neighbor classes. Then clearly an empty interior means that no representation is possible. Conditions (ii) and (iv) are very similar. Condition (iv) means that considering classes $j$ and $j^{\prime}$, we need at least $j-j^{\prime}+1$ degrees on the scale between the leftmost degree of the interior of $A_{j^{\prime}}$ and the rightmost degree of the interior of $A_{j}$ (that is, the allowable range to score all alternatives from classes $j^{\prime}, j^{\prime}+1, \ldots, j$ ). Condition (ii) is similar with the cores. It is not difficult to see that if there exists a nonempty core, then this is the allowable range for overall scores of that class. Hence if cores $K_{j}, K_{j^{\prime}}$ are both nonempty, all classes $j^{\prime}+1, \ldots, j-1$ have to fit in the space left between the two cores.

We turn now to the case where no partitioning of $E$ is needed.

Theorem 2 Let $(A, \succsim, E)$ be a decision profile with $k^{\prime}$ indifference classes. It exists a representation of $(A, \succsim, E)$ by a weak mean operator (resp. a mean operator, a strong mean, a strict mean) $G$, if and only if the conditions (i) to (iv) of Theorem 1 are satisfied, and in addition

(v) $\left|K_{j}\right| \leq 1, j=1, \ldots, k^{\prime}$.

Remark that the additional condition (v) means that in an indifference class, the pairwise intersection of alternatives is never empty.

\subsection{Representation with a refinement of the scale}

From Theorem 1, we understand that the scale $E$ has not enough degrees if one the conditions (ii), (iii) or (iv) is not fulfilled. Clearly, it suffices to add a finite number of 
new degrees to $E$ to satisfy conditions (ii) through (iv). The exact places where these degrees should be inserted, as well as the minimal number of additional degrees depend on which conditions are violated and how. We give in Section 4 an algorithm solving this problem.

Let us denote by $\bar{E}$ the refined scale. Thus, $G$ maps $E^{n}$ to $\bar{E}$, and $f$ performs a partitioning of $\bar{E}$. A direct adaptation of theorems 1 and 2 leads to the following.

Corollary 1 Let $(A, \succsim, E)$ be a decision profile, E being a finite chain.

(i) There exists a representation of $\succsim$ by $f \circ G$, where $G$ is weak mean operator (resp. a mean operator, a strong mean, a strict mean) valued on $\bar{E}, f$ defines a partition of $\bar{E}$, and $\bar{E}$ is a finite refinement of $E$, if and only if the profile is weakly coherent (resp. coherent, strongly coherent, strictly coherent).

(ii) There exists a representation of $\succsim$ by $G$, where $G$ is weak mean operator (resp. a mean operator, a strong mean, a strict mean) valued on $\bar{E}$, where $\bar{E}$ is a finite refinement of $E$, if and only if the profile is weakly coherent (resp. coherent, strongly coherent, strictly coherent), and for any indifference class $A_{j}$, any $a, b \in A_{j}, a$ and $b$ have no disjoint spans.

\section{Algorithms}

\subsection{Construction of $f$ and $G$}

The proof of Theorem 1 being constructive, it is possible to construct all possible operators $G$ and functions $f$ solving the problem. The following algorithm performs this.

\section{Algorithm constructing $f$ and $G$ (Algorithm 1)}

(1) Construction of $f$ (or equivalently $E^{\prime}$ ). For all indifference classes $A_{j}$, $j=1, \ldots, k^{\prime}$, do:

- if $\left|K_{j}\right|>1, f\left(x_{j}\right):=e_{j}^{\prime}$ for all $x_{j} \in K_{j}$ (in terms of $E^{\prime}$ : all degrees in $K_{j}$ are shrunk into a single element $e_{j}^{\prime}$ ),

- otherwise $f=I d$ (no change for $E$ )

(2) Construction of intervals for $G$.

(2.1) Initialisation. For all indifference classes $A_{j}, j=1, \ldots, k^{\prime}$, do:

$*$ if $K_{j} \neq \emptyset$, set $\left[\psi_{j}^{0}, \phi_{j}^{0}\right]=K_{j}$.

* otherwise, choose $e^{j} \in \AA_{j}$ such that:

- if $K_{j+1} \neq \emptyset$, $e^{j}<\operatorname{left}\left(K_{j+1}\right)$, otherwise choose $e^{j+1}$ in $\AA_{j+1}$ (if not already done) such that $e^{j}<e^{j+1}$

- if $K_{j-1} \neq \emptyset$, $e^{j}>\operatorname{left}\left(K_{j-1}\right)$, otherwise choose $e^{j-1}$ in $\AA_{j-1}$ (if not already done) such that $e^{j}>e^{j-1}$

and set $\left[\psi_{j}^{0}, \phi_{j}^{0}\right]=\left\{e^{j}\right\}$. 
(2.2) Construction of the intervals. For all indifference classes $A_{j}$, $j=1, \ldots, k^{\prime}$, set:

$$
\left.\left[\psi_{j}^{1}, \phi_{j}^{1}\right]=\right] \phi_{j-1}^{0}, \psi_{j+1}^{0}\left[\cap \stackrel{\circ}{A}_{j}\right.
$$

If $\exists j$ such that $\left[\psi_{j}^{1}, \phi_{j}^{1}\right] \cap\left[\psi_{j+1}^{1}, \phi_{j+1}^{1}\right]=: B_{j} \neq \emptyset$, partition $B_{j}$ in two parts, assign left part to $\left[\psi_{j}^{1}, \phi_{j}^{1}\right]$ and right part to $\left[\psi_{j+1}^{1}, \phi_{j+1}^{1}\right]$. This defines $\left[\psi_{j}, \phi_{j}\right]$, which are all pairwise disjoint.

(3) Construction of $G$. For all indifference classes $A_{j}, j=1, \ldots, k^{\prime}$, do:

$*$ if $K_{j} \neq \emptyset$ : case of:

- weak mean: for every $a \in A_{j}$, choose $G(a)$ in $K_{j} \cap \operatorname{span}(a)$

- mean: for every $a \in A_{j}$, choose $G(a)$ in $K_{j} \cap \operatorname{span}(a)$ such that for any $a, b \in A_{j}, a \geq b$ implies $G(a) \geq G(b)$

- strong mean: for every $a \in A_{j}$, choose $G(a)$ in $K_{j} \cap \operatorname{span}(a)$ such that for any $a, b \in A_{j}, a \geq b$ implies $G(a) \geq G(b)$, and $a_{i}>b_{i}$ for $i=1, \ldots, n$ imply $G(a)>G(b)$

- strict mean: for every $a \in A_{j}$, choose $G(a)$ in $K_{j} \cap \operatorname{span}(a)$ such that for any $a, b \in A_{j}, a \geq b$ implies $G(a) \geq G(b)$, with strict inequality if $a_{i}>b_{i}$ for some $i \in\{1, \ldots, n\}$.

* otherwise choose $e^{j}$ in $\left[\psi_{j}, \phi_{j}\right] \cap \bigcap_{a \in A_{j}} \operatorname{span}(a)$, and let

$$
G(a)=e^{j}, \forall a \in A_{j} .
$$

\subsection{Refinement of the scale}

Of course, infinitely many refinements of $E$ exist, and we are interested in finding the ones satisfying the conditions with the least number of degrees. A partial order can be defined on the set of refinements of a given scale $E$, denoted by $\sqsupseteq$. Let us denote by $\mathcal{E}$ the set of refinements of $E$.

Definition 5 Let $\bar{E}^{1}$ and $\bar{E}^{2}$ be in $\mathcal{E}$. Then $\bar{E}^{1} \sqsupseteq \bar{E}^{2}$ if $\bar{E}^{1}$ is a refinement of $\bar{E}^{2}$.

We propose now an algorithm for finding a refinement of $E$, when the decision profile does not fulfill conditions (ii), (iii) or (iv), but is at least a weakly coherent decision profile. The idea is to satisfy at first all conditions where only a unique smallest refinement exists. The algorithm is described below. We use the notation $\uparrow e_{i}$ to denote the successor of $e_{i}$ on the current scale, i.e., $e_{i+1}$, while $\downarrow e_{i}$ denotes its predecessor.

\section{Algorithm of refinement (Algorithm 2)}

(1) If $\exists j$ such that $\AA_{j}=\emptyset$, add a degree between $\max _{a \prec A_{j}} \operatorname{left}(a)$ and its successor. Repeat until no interior is empty.

(2.0) Check if any condition (ii) or (iv) is violated on the current scale $\bar{E}$ (i.e., cores and interiors have to be recomputed if the scale has changed). If not, stop. 
(2.1) For any violated condition (ii) or (iv) on the current refined scale $\bar{E}$, involving indices $j, j^{\prime}$ with $j^{\prime}<j$, we denote by $\left[q_{j^{\prime}}, r_{j}\right]$ the interval of $\bar{E}$ where $m_{j, j^{\prime}}$ degrees have to be added. Specifically:

- for condition (ii),

$$
q_{j^{\prime}}=\operatorname{right}\left(K_{j^{\prime}}\right), \quad r_{j}=\operatorname{left}\left(K_{j}\right), \text { and } m_{j, j^{\prime}}=j-j^{\prime}-d\left(K_{j^{\prime}}, K_{j}\right)
$$

- for condition (iv),

$$
\begin{aligned}
& q_{j^{\prime}}=\max \left(\uparrow \max _{a \prec A_{j^{\prime}}} \operatorname{left}(a), \operatorname{left}\left(A_{j^{\prime}}\right)\right), \quad r_{j}=\min \left(\operatorname{right}\left(A_{j}\right), \downarrow \min _{a \succ A_{j}} \operatorname{right}(a)\right), \text { and } \\
& m_{j, j^{\prime}}=j-j^{\prime}-D\left(\AA_{j^{\prime}}, \AA_{j}\right) .
\end{aligned}
$$

If $q_{j^{\prime}}=r_{j}$, then replace the interval by either $\left[\downarrow q_{j^{\prime}}, r_{j}\right]$ or $\left[q_{j^{\prime}}, \uparrow r_{j}\right]$.

Let $J$ be the set of all pairs $\left(j, j^{\prime}\right)$ such that $m_{j, j^{\prime}}>0$.

(2.2) Choose $K \subseteq J, K \neq \emptyset$, such that $\left\{\left[q_{j^{\prime}}, r_{j}\right]\right\}_{\left(j, j^{\prime}\right) \in K}$ is a maximal family (i.e., no other member can be added) of 2-overlapping intervals, i.e., such that $\left|\cap_{\left(j, j^{\prime}\right) \in K}\left[q_{j^{\prime}}, r_{j}\right]\right| \geq 2$. Then:

(i) If $\cap_{\left(j, j^{\prime}\right) \in K}\left[q_{j^{\prime}}, r_{j}\right]=\left[q_{j_{0}^{\prime}}, r_{j_{0}}\right]$ for some $\left(j_{0}, j_{0}^{\prime}\right)$ in $K$, add $m_{j_{0}, j_{0}^{\prime}}$ degrees in interval $\left[q_{j_{0}^{\prime}}, r_{j_{0}}\right]$. If there are several such $\left(j_{0}, j_{0}^{\prime}\right)$, take the max of $m_{j_{0}, j_{0}^{\prime}}$ over them.

(ii) Otherwise, add $m_{K}:=\min _{\left(j, j^{\prime}\right) \in K} m_{j, j^{\prime}}$ degrees in interval $\cap_{\left(j, j^{\prime}\right) \in K}\left[q_{j^{\prime}}, r_{j}\right]$.

Return to step (2.0)

Remark that the way to add degrees in step (2.2) may be not unique, if the interval contains more than 2 elements.

Theorem 3 The above algorithm permits to satisfy all violated conditions.

(see proof in appendix)

Although the algorithm produces a smallest refinement in the sense of $\sqsupseteq$ most of the time, it may happen in some situations that it is not the case, i.e., it is possible to withdraw some of the degrees of the refined scale without violating the conditions. This is shown on the following example.

ExAmple 3: Assume $E=\left\{e_{1}, \ldots, e_{8}\right\}$, and violated conditions lead to the following table of demands:

\begin{tabular}{|c|c|}
\hline$\left[q_{j, j^{\prime}}, r_{j, j^{\prime}}\right]$ & $m_{j, j^{\prime}}$ \\
\hline$\left[e_{1}, e_{4}\right]$ & 1 \\
{$\left[e_{2}, e_{5}\right]$} & 1 \\
{$\left[e_{3}, e_{5}\right]$} & 1 \\
{$\left[e_{4}, e_{7}\right]$} & 1 \\
{$\left[e_{4}, e_{6}\right]$} & 1 \\
{$\left[e_{5}, e_{7}\right]$} & 1 \\
\hline
\end{tabular}


The maximal families are $F_{1}=\left\{\left[e_{1}, e_{4}\right],\left[e_{2}, e_{5}\right],\left[e_{3}, e_{5}\right]\right\}$, $F_{2}=\left\{\left[e_{2}, e_{5}\right],\left[e_{3}, e_{5}\right],\left[e_{4}, e_{7}\right],\left[e_{4}, e_{6}\right]\right\}$ and $F_{3}=\left\{\left[e_{4}, e_{7}\right],\left[e_{4}, e_{6}\right],\left[e_{5}, e_{7}\right]\right\}$. In Step (2.2), a natural choice may be to choose the largest maximal family, hence $F_{2}$ in this case. Satisfying $F_{2}$ first causes the fact that $F_{1}$ and $F_{3}$ will not be satisfied, and since they are disjoint, 3 degrees are necessary. Observe however that only 2 degrees suffice if one satisfies first $F_{1}$ and then $F_{3}$.

This situation could happen every time there is a proper inclusion of a maximal family $\left(F_{2}\right)$ into the union of two others $\left(F_{1}, F_{3}\right)$.

This shows that the choice of the sequence of maximal families to be satisfied is important, and is not a trivial problem. A way to make the algorithm minimal in the sense of $\sqsupseteq$ is to add a last step where it is checked that all new degrees are indeed necessary to satisfy the conditions.

We illustrate the whole process on two practical examples, using an implementation in $\mathrm{C}++$ of the above algorithms. In both examples we consider a list of students, who are evaluated on 3 subjects (say mathematics, physics and literature), on a qualitative scale $E$ with 4 or 5 degrees. The degrees of the scale will be simply referred to by their number, with 1 being the worst degree and 5 (or 4 ) the best one.

ExAmple 4: We consider 6 students $A, B, C, D, E, F$ evaluated on a scale of 4 degrees. Table 1 gives their score. The ranking determined by the teachers

\begin{tabular}{|c||c|c|c|}
\hline Student & Math. & Physics & Liter. \\
\hline A & 2 & 1 & 1 \\
B & 1 & 1 & 3 \\
C & 1 & 4 & 2 \\
D & 3 & 4 & 2 \\
E & 2 & 3 & 4 \\
F & 3 & 4 & 4 \\
\hline
\end{tabular}

Table 1: Scores obtained by the 6 students

is simply $A \prec B \prec C \prec D \prec E \prec F$, and the decision profile is coherent. Hence there are 6 classes $A_{1}, \ldots, A_{6}$, each containing exactly one student, and obviously we need at least two additional degrees in the scale to represent the preference. Hence we start by applying Algorithm 2 to refine the scale. We remark that all cores are empty, and the interior of classes are given in Table 2. In step (2.0), we see that condition (iv) is violated. Performing step (2.1), we find the following details about the violated conditions (see Table 3). We perform step (2.2). Maximal families are

$$
\begin{aligned}
& F_{1}=\{[1,2],[1,3],[1,4]\} \\
& F_{2}=\{[1,3],[1,4],[2,3],[2,4]\} \\
& F_{3}=\{[1,4],[2,4],[3,4]\} .
\end{aligned}
$$

Let us choose $F_{1}$. Then we add one new degree in [1,2], say 1 '. We go back to (2.0) and recompute the interiors (Table 4). Results of step (2.1) are put 


\begin{tabular}{|c||c|c|}
\hline Class & Span & Interior \\
\hline$A_{1}$ & {$[1,2]$} & {$[1,2]$} \\
$A_{2}$ & {$[1,3]$} & {$[2,3]$} \\
$A_{3}$ & {$[1,4]$} & {$[2,3]$} \\
$A_{4}$ & {$[2,4]$} & {$[2,3]$} \\
$A_{5}$ & {$[2,4]$} & {$[3]$} \\
$A_{6}$ & {$[3,4]$} & {$[3,4]$} \\
\hline
\end{tabular}

Table 2: Characteristics of the indifference classes

\begin{tabular}{|c|c|c|c|c|c|}
\hline$j$ & $j^{\prime}$ & $q_{j^{\prime}}$ & $r_{j}$ & {$\left[q_{j^{\prime}}, r_{j}\right]$} & $m_{j, j^{\prime}}$ \\
\hline 4 & 1 & 1 & 2 & {$[1,2]$} & 1 \\
5 & 1 & 1 & 3 & {$[1,3]$} & 2 \\
6 & 1 & 1 & 4 & {$[1,4]$} & 2 \\
4 & 2 & 2 & 2 & {$[1,2]$} & 1 \\
5 & 2 & 2 & 3 & {$[2,3]$} & 2 \\
6 & 2 & 2 & 4 & {$[2,4]$} & 2 \\
5 & 3 & 3 & 3 & {$[2,3]$} & 1 \\
6 & 3 & 3 & 4 & {$[3,4]$} & 1 \\
\hline
\end{tabular}

Table 3: Step (2.1) of Algorithm 2

in Table 5. We perform step (2.2). There is one maximal family:

$$
F_{1}=\{[1,3],[1,4],[2,3],[2,4]\}
$$

Then we add one new degree in $[2,3]$, say 2'. We go back to step (2.0) and recompute the interiors (Table 6 ). Then condition (iv) is no more violated, and the algorithm stops.

We run Algorithm 1 to compute the scores of the different classes. Since there are 6 classes and 6 degrees, the only solution is immediate:

$$
G(A)=1, \quad G(B)=1^{\prime}, \quad G(C)=2, \quad G(D)=2^{\prime}, \quad G(E)=3, \quad G(F)=4 .
$$

Let us try now to exploit this result. $G$ being specified only on the set of 6 students, it would be interesting to extend it to all possible students. To do

\begin{tabular}{|c|c|}
\hline Class & Interior \\
\hline$A_{1}$ & {$[1,2]$} \\
$A_{2}$ & {$\left[1^{\prime}, 3\right]$} \\
$A_{3}$ & {$[1,3]$} \\
$A_{4}$ & {$[2,3]$} \\
$A_{5}$ & {$[3]$} \\
$A_{6}$ & {$[3,4]$} \\
\hline
\end{tabular}

Table 4: Recomputation of the interiors 


\begin{tabular}{|c|c|c|c|c|c|}
\hline$j$ & $j^{\prime}$ & $q_{j^{\prime}}$ & $r_{j}$ & {$\left[q_{j^{\prime}}, r_{j}\right]$} & $m_{j, j^{\prime}}$ \\
\hline 5 & 1 & 1 & 3 & {$[1,3]$} & 1 \\
6 & 1 & 1 & 4 & {$[1,4]$} & 1 \\
5 & 2 & 2 & 3 & {$[2,3]$} & 1 \\
6 & 2 & 2 & 4 & {$[2,4]$} & 1 \\
\hline
\end{tabular}

Table 5: Step (2.1) of Algorithm 2

\begin{tabular}{|c|c|}
\hline Class & Interior \\
\hline$A_{1}$ & {$[1,2]$} \\
$A_{2}$ & {$\left[1^{\prime}, 3\right]$} \\
$A_{3}$ & {$\left[1^{\prime}, 3\right]$} \\
$A_{4}$ & {$[2,3]$} \\
$A_{5}$ & {$\left[2^{\prime}, 3\right]$} \\
$A_{6}$ & {$[3,4]$} \\
\hline
\end{tabular}

Table 6: Recomputation of the interiors

this, it is convenient to choose a family of mean operators and to take in it the operator closest to the one specified by the algorithm. It is known that the Sugeno integral is a large family of operators able to deal with ordinal scales (we give here only the minimum of details. For further reference, see, e.g., Dubois et al. [2001], Grabisch and Labreuche [2008]). For a vector of scores $\left(a_{1}, \ldots, a_{n}\right)$ on the scale $E$, the Sugeno integral is computed by:

$$
\mathcal{S}_{\mu}\left(a_{1}, \ldots, a_{n}\right):=\bigvee_{i=1}^{n}\left(a_{\sigma(i)} \wedge \mu(\{\sigma(i), \ldots, \sigma(n)\})\right)
$$

where $\sigma$ is a permutation on $\{1, \ldots, n\}$ rearranging the scores in increasing order, i.e., $a_{\sigma(1)} \leq \cdots \leq a_{\sigma(n)}, \vee, \wedge$ indicate maximum and minimum, and $\mu$ is a capacity, i.e., a set function defined on the set of criteria $N$, assigning to every subset of $N$ a number in $E$ representing the importance of the subset for the decision. It is easy to check that there indeed exists a capacity $\mu$ so that $\mathcal{S}_{\mu}$ coincide with $G$ on the 6 students. It is given in Table 7 , where M, P and L stand for Maths, Physics and Literature respectively. Using $\mathcal{S}_{\mu}$, every

\begin{tabular}{|c|ccccccc|}
\hline subset $A$ & $\mathrm{M}$ & $\mathrm{P}$ & $\mathrm{L}$ & $\mathrm{M}, \mathrm{P}$ & $\mathrm{M}, \mathrm{L}$ & $\mathrm{P}, \mathrm{L}$ & $\mathrm{M}, \mathrm{P}, \mathrm{L}$ \\
\hline$\mu(A)$ & 1 & 2 & $1^{\prime}$ & $2^{\prime}$ & $1^{\prime}$ & 4 & 4 \\
\hline
\end{tabular}

Table 7: Definition of the capacity $\mu$

student can be scored, and students $\mathrm{A}$ to $\mathrm{F}$ will receive the same score as the one given by $G$. Table 8 gives some examples.

ExAmple 5: We consider 11 students $A, \ldots, K$ evaluated on a scale of 5 degrees. Table 9 gives their scores. The following ranking has been determined 


\begin{tabular}{|c|ccc|c|}
\hline student & M & P & L & overall score \\
\hline G & 4 & 1 & 2 & 1 \\
H & 2 & 4 & 1 & 2 \\
I & 4 & 3 & 1 & $2^{\prime}$ \\
J & 2 & 1 & 4 & 1 \\
$\vdots$ & $\vdots$ & $\vdots$ & $\vdots$ & $\vdots$ \\
\hline
\end{tabular}

Table 8: Scoring of new students

\begin{tabular}{|c||c|c|c|}
\hline Student & Math. & Physics & Liter. \\
\hline $\mathrm{A}$ & 1 & 1 & 3 \\
$\mathrm{~B}$ & 1 & 2 & 1 \\
\hline $\mathrm{C}$ & 1 & 3 & 2 \\
\hline $\mathrm{D}$ & 2 & 2 & 2 \\
$\mathrm{E}$ & 3 & 2 & 2 \\
$\mathrm{~F}$ & 2 & 2 & 5 \\
\hline $\mathrm{G}$ & 4 & 4 & 4 \\
$\mathrm{H}$ & 4 & 4 & 3 \\
\hline $\mathrm{I}$ & 5 & 3 & 3 \\
\hline $\mathrm{J}$ & 4 & 3 & 5 \\
\hline $\mathrm{K}$ & 5 & 3 & 5 \\
\hline
\end{tabular}

Table 9: Scores obtained by the 11 students

by the teachers:

$$
A \sim B \prec C \prec D \sim E \sim F \prec G \sim H \prec I \prec J \prec K .
$$

They are 7 indifference classes, labelled from 1 (worst) to 7 (best). One can verify that the decision profile is coherent. Table 10 gives the span, cores and interiors of all classes. Obviously conditions of Th. 2 are not satisfied

\begin{tabular}{|c||c|c|c|}
\hline Class & Span & Core & Interior \\
\hline 1 & {$[1,3]$} & empty & {$[1,1]$} \\
2 & {$[1,3]$} & empty & empty \\
3 & {$[2,5]$} & {$[2,2]$} & {$[2,3]$} \\
4 & {$[3,4]$} & {$[4,4]$} & {$[3,4]$} \\
5 & {$[3,5]$} & empty & empty \\
6 & {$[3,5]$} & empty & empty \\
7 & {$[3,5]$} & empty & {$[5,5]$} \\
\hline
\end{tabular}

Table 10: Characteristics of the indifference classes

since there are empty interiors, hence a refinement is necessary. However, since cores are reduced to singletons, no shrinking of the scale is necessary. 
Application of the refinement algorithm (Algorithm 2) leads to add 3 new degrees as follows (new degrees are denoted with' and "):

$$
1<1^{\prime}<2<3<4<4^{\prime}<4^{\prime \prime}<5 \text {. }
$$

The application of Algorithm 1 on this new scale $\bar{E}$ leads to a unique solution for $G$ :

$$
\begin{gathered}
G(\mathrm{~A})=G(\mathrm{~B})=1, G(\mathrm{C})=1^{\prime}, G(\mathrm{D})=G(\mathrm{E})=G(\mathrm{~F})=2, G(\mathrm{G})=G(\mathrm{H})=4, \\
G(\mathrm{I})=4^{\prime}, G(\mathrm{~J})=4^{\prime \prime}, G(K)=5 .
\end{gathered}
$$

We give some details on how $G$ has been obtained by Algorithm 1. First, we recompute the interiors of the classes on the new scale (cores and spans have not changed). This is given in Table 11. Step 1 is void (no shrinking of

\begin{tabular}{|c|c|}
\hline Class & Interior \\
\hline 1 & {$\left[1,1^{\prime}\right]$} \\
2 & {$\left[1^{\prime}, 1^{\prime}\right]$} \\
3 & {$[2,3]$} \\
4 & {$[3,4]$} \\
5 & {$\left[4^{\prime}, 4^{\prime \prime}\right]$} \\
6 & {$\left[4^{\prime}, 4^{\prime \prime}\right]$} \\
7 & {$\left[4^{\prime}, 5\right]$} \\
\hline
\end{tabular}

Table 11: Interior of classes

the scale). Steps (2.1) and (2.2) build intervals $\left[\psi_{j}^{0}, \phi_{j}^{0}\right],\left[\psi_{j}^{1}, \phi_{j}^{1}\right]$ and $\left[\psi_{j}, \phi_{j}\right]$. Table 12 gives the intervals computed in these steps. In step 3, the mean

\begin{tabular}{|c|ccc|}
\hline class $A_{j}$ & {$\left[\psi_{j}^{0}, \phi_{j}^{0}\right]$} & {$\left[\psi_{j}^{1}, \phi_{j}^{1}\right]$} & {$\left[\psi_{j}, \phi_{j}\right]$} \\
\hline$A_{1}$ & {$[1,1]$} & {$[1,1]$} & {$[1,1]$} \\
$A_{2}$ & {$\left[1^{\prime}, 1^{\prime}\right]$} & {$\left[1^{\prime}, 1^{\prime}\right]$} & {$\left[1^{\prime}, 1^{\prime}\right]$} \\
$A_{3}$ & {$[2,2]$} & {$[2,3]$} & {$[2,3]$} \\
$A_{4}$ & {$[4,4]$} & {$[3,4]$} & {$[4,4]$} \\
$A_{5}$ & {$\left[4^{\prime}, 4^{\prime}\right]$} & {$\left[4^{\prime}, 4^{\prime}\right]$} & {$\left[4^{\prime}, 4^{\prime}\right]$} \\
$A_{6}$ & {$\left[4^{\prime \prime}, 4^{\prime \prime}\right]$} & {$\left[4^{\prime \prime}, 4^{\prime \prime}\right]$} & {$\left[4^{\prime \prime}, 4^{\prime \prime}\right]$} \\
$A_{7}$ & {$[5,5]$} & {$[5,5]$} & {$[5,5]$} \\
\hline
\end{tabular}

Table 12: Intervals for classes computed by Algorithm 1

operator is constructed, and there is only one possibility.

Let us try as in Example 4 to extend the definition of $G$ to every possible student. If one wants to find a capacity so that the Sugeno integral is an extension of $G$, it leads to contradictory constraints on the capacity, so that no Sugeno integral can represent the preference. Indeed, computing the score 
of students B and C leads to:

$$
\begin{aligned}
1 & =\mathcal{S}_{\mu}(1,2,1)=(1 \wedge \mu(\{M, P, L\})) \vee(2 \wedge \mu(\{P\})) \Rightarrow \mu(\{P\})=1 . \\
1^{\prime} & =\mathcal{S}_{\mu}(1,3,2)=(1 \wedge \mu(\{M, P, L\})) \vee(2 \wedge \mu(\{P, L\})) \vee(3 \wedge \mu(\{P\})) \Rightarrow \mu(\{P\})=1^{\prime} .
\end{aligned}
$$

Then other methods have to be used. For example, the rule-based method of Greco et al. could be used (see Greco et al. [2001, 2004, 2005]). From a table of data like Table 9 , together with the overall score computed by $G$, it is possible to construct a set of rules compatible with the data.

\section{Conclusion}

We have provided a general method with practical algorithms for scoring alternatives, when they are evaluated on some common ordinal scale. The method is able to deal with various conditions of coherence on the decision profile, including the very weak condition of weak coherence, and its originality is to build an adequate scale for representing the overall scores.

Specifically, the two algorithms presented in the paper permit to:

- check if the preference is representable by a mean operator (weak, strong, strict,...), given the scale.

- refine the scale if necessary, adding new degrees where it is necessary. All possible solutions are provided for the new scale.

- if the preference is representable, perform if necessary a shrinking of the scale, then construct a mean operator (weak, strong, strict,...) on the set of alternatives. Again, all possible solutions are provided.

Although the method can be used as it is, just producing a scoring of a given set of alternatives, it can be used as a first step in building a model, which could then rank and score other alternatives, without specifying the preferences of the decision maker. For this, it is necessary to extend the definition of $G$, which is known only on the original set of alternatives $A$, to the whole potential set of alternatives $E^{n}$. This extension viewed as a general problem is a challenging aim for further research. However, as shown in Example 4 and Example 5, there exist already some methods to deal with this problem. In Example 4, the Sugeno integral has been applied. In Example 5, we suggest the rulebased approach of Greco et al. Finally, we would like to mention a real-sized application of this method by Jullien et al. [2006].

\section{References}

C. A. Bana e Costa and J.-C. Vansnick. A theoretical framework for Measuring Attractiveness by a Categorical Based Evaluation TecHnique (MACBETH). In Proc. XIth Int. Conf. on MultiCriteria Decision Making, pages 15-24, Coimbra, Portugal, August 1994. 
C. A. Bana e Costa and J.-C. Vansnick. Applications of the MACBETH approach in the framework of an additive aggregation model. J. of Multicriteria Decision Analysis, 6: 107-114, 1997.

J.-P. Brans and P. Vincke. A preference ranking organization method. Management Science, 31:647-656, 1985.

D. Dubois, J.-L. Marichal, H. Prade, M. Roubens, and R. Sabbadin. The use of the discrete Sugeno integral in decision making: a survey. Int. J. of Uncertainty, Fuzziness and Knowledge-Based Systems, 9(5):539-561, 2001.

J. Fodor. Smooth associative operations on finite ordinal scales. IEEE Tr. on Fuzzy Systems, 8(6):791-795, 2000.

M. Grabisch. Representation of preferences over a finite scale by a mean operator. Math. Social Sciences, 52:131-151, 2006.

M. Grabisch and Ch. Labreuche. A decade of application of the Choquet and Sugeno integrals in multi-criteria decision aid. 4OR, 6:1-44, 2008. doi 10.1007/s10288-0070064-2.

S. Greco, B. Matarazzo, and R. Słowiński. Rough sets theory for multicriteria decision analysis. Eur. J. of Opertional Research, 129:1-47, 2001.

S. Greco, B. Matarazzo, and R. Słowiński. Axiomatic characterization of a general utility function and its particular cases in terms of conjoint measurement and rough-set decision rules. Eur. J. of Operational Research, 158(2):271-292, 2004.

S. Greco, B. Matarazzo, and R. Słowiński. Decision rule approach. In J. Figueira, S. Greco, and M. Ehrgott, editors, Multiple Criteria Decision Analysis, pages 507-561. Kluwer Academic Publishers, 2005.

S. Jullien, G. Mauris, L. Valet, and P. Bolon. Decision aiding tools for animated film selection from a mean aggregation of criteria preferences over a finite scale. In Proc. Int. Conf. on Information Processing and Management of Uncertainty (IPMU'06), pages 643-650, Paris, France, July 2006.

J.-L. Marichal. On Sugeno integral as an aggregation function. Fuzzy Sets and Systems, 114:347-365, 2000.

J.-L. Marichal and R. Mesiar. Aggregation on finite ordinal scales by scale independent functions. Order, 21:155-180, 2004.

J.-L. Marichal, R. Mesiar, and T. Rückschlossová. A complete description of comparison meaningful functions. Aequationes Mathematicae, 69:309-320, 2005a.

J.-L. Marichal, P. Meyer, and M. Roubens. Sorting multi-attribute alternatives: the TOMASO method. Computers \& Operations Research, 32:861-877, 2005b.

M. Mas, G. Mayor, and J. Torrens. T-operators and uninorms on a finite totally ordered set. Int. J. Intelligent Systems, 14(9):909-922, 1999. 
M. Mas, M. Monserrat, and J. Torrens. On bisymmetric operators on a finite chain. IEEE Tr. on Fuzzy Systems, 11(5):647-651, 2003.

B. Massam and I. Askew. Methods for comparing policies using multiple criteria: an urban example. OMEGA, 10(2):195-204, 1982.

P. Meyer and M. Roubens. Choice, ranking and sorting in fuzzy multiple criteria decision aid. In J. Figueira, S. Greco, and M. Ehrgott, editors, Multiple Criteria Decision Analysis, pages 471-506. Kluwer Academic Publishers, 2005.

S. Ovchinnikov. Means on ordered sets. Mathematical Social Sciences, 32:39-56, 1996.

J. Paelinck. Qualitative multiple criteria analysis, environmental protection and multiregional development. Papers of the Regional Science Association, 36:59-74, 1976.

M. Pirlot and Ph. Vincke. Lexicographic aggregation of semiorders. J. of Multi-Criteria Decision Analysis, 1:47-58, 1992.

J.-C. Pomerol and S. Barba-Romero. Multicriterion decision in management: principles and practice. Kluwer Academic Publishers, 2000.

A. Rico, M. Grabisch, Ch. Labreuche, and A. Chateauneuf. Preference modelling on totally ordered sets by the Sugeno integral. Discrete Applied Mathematics, 147:113$124,2005$.

F. S. Roberts. Measurement Theory. Addison-Wesley, 1979.

B. Roy. Classement et choix en présence de points de vue multiples (la méthode ELECTRE). R.I.R.O., 2:57-75, 1968.

B. Roy. How outranking relations helps multiple criteria decision making. In J.-L. Cochrane and M. Zeleny, editors, Multiple Criteria Decision Making, pages 179-201. University of South California Press, Columbia, 1973.

M. Sugeno. Theory of fuzzy integrals and its applications. PhD thesis, Tokyo Institute of Technology, 1974.

J.-C. Vansnick. On the problem of weights in multiple criteria decision making, the noncompensatory approach. Eur. J. of Operational Research, 24:288-294, 1986.

\section{A Proof of Theorem 3}

We need for this purpose two elementary results.

Lemma 1 Let $(A, \succsim, E)$ be a weakly coherent decision profile. Then the following holds.

(i) The non empty cores (if any) are disjoint, and ordered the right way, i.e., $K_{j}$ is to the left of $K_{j^{\prime}}$ if and only if $j^{\prime}<j$.

(ii) For any class $A_{j}, \max _{a \prec A_{j}} \operatorname{left}(a)<\min _{a \succ A_{j}} \operatorname{right}(a)$. 
These results are respectively Lemma 3 and Lemma 4 (i) in Grabisch [2006]. For the sake of completeness and since they are very easy to prove, we give the proof below.

Proof: (i) Suppose $K_{j^{\prime}} \cap K_{j} \neq \emptyset, j>j^{\prime}$, so that there exists say $e_{l} \in E$ in the intersection. Since $e_{l} \in K_{j^{\prime}}$, there exist $a, b \in A_{j^{\prime}}$ such that $\operatorname{right}(a) \leq e_{l} \leq \operatorname{left}(b)$. Similarly, since $e_{l} \in K_{j}$, there exist $c, d \in A_{j}$ such that $\operatorname{right}(c) \leq e_{l} \leq \operatorname{left}(d)$. This entails right $(c) \leq \operatorname{left}(b)$. But by definition of $A_{j^{\prime}}, A_{j}$, we have $c \succ b$, which violates weak coherence. Now, $K_{j^{\prime}}$ is to the left of $K_{j}$, otherwise weak coherence will be clearly violated too.

(ii) Let us denote by $a^{>j}$ and $a^{<j}$ the alternatives (possibly non unique) such that $\operatorname{right}\left(a^{>j}\right)=\min _{a \succ A_{j}} \operatorname{right}(a)$, and left $\left(a^{<j}\right)=\max _{a \prec A_{j}} \operatorname{right}(a)$.

Suppose that $\max _{a \prec A_{j}} \operatorname{left}(a) \geq \min _{a \succ A_{j}} \operatorname{right}(a)$. This implies that $\operatorname{left}\left(a^{<j}\right) \geq \operatorname{right}\left(a^{>j}\right)$. But $a^{<j} \prec a^{>j}$, and the weak coherence assumption is violated. Thus, $\max _{a \prec A_{j}} \operatorname{left}(a)<$ $\min _{a \succ A_{j}} \operatorname{right}(a)$.

Proof: (of Theorem 3) We prove that the successive steps make the conditions to be satisfied.

(1) From the condition of weak coherence and Lemma 1 (ii), we know that $\AA_{j}=\emptyset$ is equivalent to $\left|\left[\max _{a \prec A_{j}} \operatorname{left}(a), \min _{a \succ A_{j}} \operatorname{right}(a)\right]\right|=2$. So it suffices to insert one new degree between these bounds to make $\AA_{j} \neq \emptyset$.

(2) Remark that it is impossible to split an interval which is a singleton. Hence, this case has to be avoided. Due to Lemma 1 (i), this cannot be the case with cores (condition (ii)). However, this may occur for interiors. Due to the definition, a singleton occurs when $\max \left(\uparrow \max _{a \prec A_{j^{\prime}}} \operatorname{left}(a), \operatorname{left}\left(A_{j^{\prime}}\right)\right)=\min \left(\operatorname{right}\left(A_{j}\right), \downarrow \min _{a \succ A_{j}} \operatorname{right}(a)\right)=: e_{i_{0}}$ for some $e_{i_{0}} \in E$. Then necessarily, $\max _{a \prec A_{j^{\prime}}} \operatorname{left}(a)<e_{i_{0}}<\min _{a \succ A_{j}} \operatorname{right}(a)$. Inserting degrees in $\left[\downarrow e_{i_{0}}, e_{i_{0}}\right]$, or $\left[e_{i_{0}}, \uparrow e_{i_{0}}\right]$ will correct the violated condition, unless both spans $\operatorname{span}\left(A_{j^{\prime}}\right), \operatorname{span}\left(A_{j}\right)$ are reduced to $\left\{e_{i_{0}}\right\}$. But this is impossible since the profile is weakly coherent. Now, if both supports are to the left of $\uparrow e_{i_{0}}$ (resp. right of $\downarrow e_{i_{0}}$ ), add degrees to the left (resp. to the right) of $e_{i_{0}}$.

Thanks to this convention, we can assume that any interval $\left[q_{j^{\prime}}, r_{j}\right]$ contains at least two elements.

Let us show that the algorithm corrects violated conditions. First, for condition $2.2(\mathrm{i})$, remark that adding $m_{j, j^{\prime}}$ degrees in $\left[q_{j^{\prime}}, r_{j}\right]$ makes the violated condition to be satisfied. Second, for condition 2.2(ii), adding $m_{K}$ degrees in an intersection of intervals $\left\{\left[q_{j^{\prime}}, r_{j}\right]\right\}_{\left(j, j^{\prime}\right) \in K}$ decreases the quantities $m_{j, j^{\prime}}$ by $m_{K}$, for any $\left(j, j^{\prime}\right) \in K$. Since $m_{K}=$ $\min _{\left(j, j^{\prime}\right) \in K} m_{j, j^{\prime}}$, in each step at least one violated condition becomes satisfied. Hence, in a finite number of steps, no more violated condition will remain. 